\title{
Crystal-Field Interactions in $\mathrm{RPd}_{2} \mathrm{Al}_{3}$ Intermetallics $(\mathrm{R}=\mathrm{Pr}, \mathrm{Nd}, \mathrm{Sm}$, and $\mathrm{U})$
}

\author{
R.J. RADWANSKI ${ }^{a, b, *}$, D.M. NALECZ ${ }^{b}$ AND Z. ROPKA ${ }^{a}$ \\ ${ }^{a}$ Center of Solid State Physics, św. Filip 5, 31-150 Kraków, Poland \\ ${ }^{b}$ Institute of Physics, Pedagogical University, 30-084 Kraków, Poland
}

\begin{abstract}
Magnetic and electronic properties of $\mathrm{NdPd}_{2} \mathrm{Al}_{3}$ have been nicely described with the $\mathrm{Nd}^{3+}$ ions within the crystal-field theory revealing that all $\mathrm{Nd}$ atoms equally contribute to the temperature dependence of the paramagnetic susceptibility and of the heat capacity with the $\lambda$-peak. We consistently described paramagnetic and magnetically-ordered state. The fine electronic structure of the $\mathrm{Nd}^{3+}$ ion nicely correlates with the electronic structure of the $\mathrm{U}^{3+}$ ion in $\mathrm{UPd}_{2} \mathrm{Al}_{3}$ giving strong evidence for the trivalent uranium state in the isostructural heavy-fermion superconductor $\mathrm{UPd}_{2} \mathrm{Al}_{3}$.
\end{abstract}

DOI: 10.12693/APhysPolA.126.A-32

PACS: 71.70.Ej, 75.10.Dg

\section{Introduction}

The description of electronic and magnetic properties of $\mathrm{RPd}_{2} \mathrm{Al}_{3}$ has started in 1993 [1] just after the first preparation of $\mathrm{UPd}_{2} \mathrm{Al}_{3}$ in $1991[2]$ and the discovery in this uranium intermetallic compound both the heavy-fermion (h-f) behavior and the superconductivity $[3,4]$. Studies of $\mathrm{RPd}_{2} \mathrm{Al}_{3}$ intermetallics have been, somehow, motivated by a physical idea to help in understanding of these exotic properties of $\mathrm{UPd}_{2} \mathrm{Al}_{3}$. $\mathrm{UPd}_{2} \mathrm{Al}_{3}$ is still under hot debate though its exotic properties has been discovered already more than 20 years ago. The uniqueness of $\mathrm{UPd}_{2} \mathrm{Al}_{3}$ relies in the coexistence of the heavy-fermion phenomena and the large magnetic moment of about $0.85-1.5 \mu_{\mathrm{B}}$, below $T_{\mathrm{N}}=14.3 \mathrm{~K}$, as well as the superconductivity below $2 \mathrm{~K}$. The main point of the debate is related to the understanding of the role played by $f$ electrons - they are localized or itinerant, or more exactly how many $f$ electrons are localized or itinerant $[2,3,5-7]$.

In this paper we have re-analyzed experimental results on $\mathrm{NdPd}_{2} \mathrm{Al}_{3}$ [8]. We have confirmed the crystal-field (CEF) parameters obtained by the Furrer group as well as the derived fine electronic structure (FES) with the attributing eigenfunctions. We have derived properties of $\mathrm{NdPd}_{2} \mathrm{Al}_{3}$ compound in the (antiferro-)magnetic state and make crystal-field theory predictions for the magnetic and electronic properties of $\mathrm{RPd}_{2} \mathrm{Al}_{3}(\mathrm{R}=\mathrm{Pr}$, $\mathrm{Ce}$, and $\mathrm{Sm}$ ). Finally, we will compare the FES of the $\mathrm{Nd}^{3+}$ ion in $\mathrm{NdPd}_{2} \mathrm{Al}_{3}$ with obtained earlier by us FES of the $\mathrm{U}^{3+}$ ion in $\mathrm{UPd}_{2} \mathrm{Al}_{3}$ - their similarity points to our long-time claim for the trivalent uranium state in $\mathrm{UPd}_{2} \mathrm{Al}_{3}[7]$.

\section{Theoretical outline}

For the description of electronic and magnetic properties of a compound containing rare-earth or actinide

${ }^{*}$ corresponding author; e-mail: sfradwan@cyf-kr.edu.pl atom (in fact, also of a $3 d$ atom) we start from a detailed analysis of conventional interactions. Such analysis starts with a trial attributing the integer valency to the involved $4 f / 5 f / 3 d$ atoms and subsequently we assume that the $4 f / 5 f / 3 d$ ions keep their atomic-like integrity also being a part of the crystal lattice (thus we call our approach the quantum atomistic solid-state (QUASST) theory [9]). Being specific, we think that $\mathrm{Nd}$ atoms, for instance, in $\mathrm{NdPd}_{2} \mathrm{Al}_{3}$ become $\mathrm{Nd}^{3+}$ ions with the $4 f^{3}$ configuration maintaining its characteristics known from the atomic physics. A consequence of such approach is that for the $4 f / 5 f$ ions we assume that the intra-atomic relativistic spin-orbit interaction is sufficiently strong - it means that the total $J$ becomes the good quantum number and that the higher multiplets are so high in energy that their population even in the room and ambient temperatures is negligibly small. So, we have applied a single-ion like Hamiltonian for the ground multiplet ${ }^{4} I_{9 / 2}(J=9 / 2$ for the $\mathrm{Nd}^{3+}$ and $\mathrm{U}^{3+}$ ions) completed with inter-site spin interactions $[7,9]$ :

$$
\begin{aligned}
& H=H_{\mathrm{CF}}+H_{f-f}= \\
& \quad \sum \sum B_{n}^{m} O_{n}^{m}+\lambda_{R R} g^{2} \mu_{\mathrm{B}}^{2}\left(-J\langle J\rangle+\frac{1}{2}\langle J\rangle^{2}\right) .
\end{aligned}
$$

The first term is the crystal-field Hamiltonian written in the well-known Stevens notation. The second term takes into account intersite spin-dependent interactions, avoiding the double counting that produces the magnetic order below $\mathrm{T}_{\mathrm{N}}$. Let us note that $-g J \mu_{\mathrm{B}}$ is the magnetic moment of the involved $\mathrm{R}$ ion, $m_{R}=-g J \mu_{\mathrm{B}}$, and that the internal molecular field is given as usually $B_{\mathrm{mol}}=\lambda_{R R} m_{R}$, with $\lambda_{R R}$ being the molecular-field coefficient.

The crystal-field Hamiltonian for the hexagonal symmetry relevant for all considered here compounds takes the form

$$
H_{\mathrm{CF}}=B_{2}^{0} O_{2}^{0}+B_{4}^{0} O_{4}^{0}+B_{6}^{0} O_{6}^{0}+B_{6}^{6} O_{6}^{6} .
$$

Under the action of these multipolar crystal-field interactions the 10 -fold degenerated ${ }^{4} I_{9 / 2}$ multiplet is split 
into 5 Kramers doublets, denoted as $\Gamma_{7}, \Gamma_{8}^{(1)}, \Gamma_{8}^{(2)}, \Gamma_{9}^{(1)}$ and $\Gamma_{9}^{(2)}$. These Kramers doublets become split in the magnetically ordered state. The appearance of this splitting manifests usually in the specific heat experiment as the $\lambda$-peak in the temperature dependence of the heat capacity at $\mathrm{T}_{\mathrm{N}}$.

\section{Results and discussion}

\section{1 $\mathrm{NdPd}_{2} \mathrm{Al}_{3}$}

A milestone in the atomic-scale theoretical description of $\mathrm{NdPd}_{2} \mathrm{Al}_{3}$ was the INS experiment in 1997 of Donni et al. [8], which revealed the crystal-field excitations with energies of $0.83,3.41$ and $8.0 \mathrm{meV}$. This very experienced group of Furrer [8] has derived a set of CEF parameters of the hexagonal symmetry for the $\mathrm{Nd}^{3+}$ ion $\left(4 f^{3}\right.$ configuration): $B_{2}^{0}=+0.214 \mathrm{meV}, B_{4}^{0}=-1.05 \mu \mathrm{eV}$, $B_{6}^{0}=-0.0224 \mu \mathrm{eV}$ and $B_{6}^{6}=+0.438 \mu \mathrm{eV}$ yielding the $\Gamma_{7}$ ground doublet. We have checked if these parameters give the same energy states as they have published in Ref. [8]. With CEF parameters collected in Table I

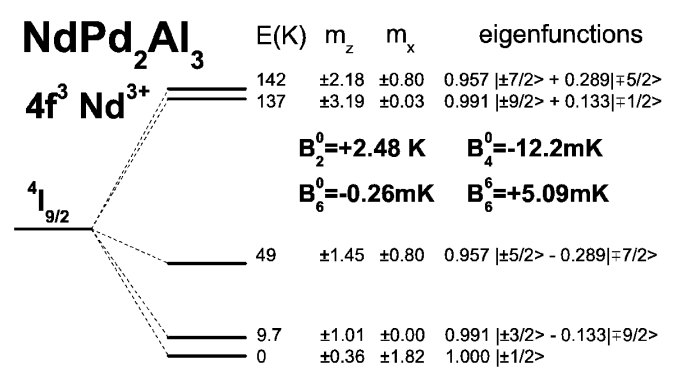

Fig. 1. Energy level scheme of the $\mathrm{Nd}^{3+}$ ion in $\mathrm{NdPd}_{2} \mathrm{Al}_{3}$ calculated with the $\mathrm{CEF}$ parameters derived in Ref. [8].

we have got exactly the same energies and exactly the same eigenfunctions by means of our computer program BIREC developed independently in our group already in year of 1990 (we customarily use the energy units of $\mathrm{K}$ ). The obtained electronic structure of $\mathrm{NdPd}_{2} \mathrm{Al}_{3}$ is shown in Fig. 1 together with eigenfunctions and magnetic characteristics. In Table I we have calculated probabilities for the transitions between the CEF levels in the inelastic-neutron-scattering on powder sample for $\mathrm{NdPd}_{2} \mathrm{Al}_{3}$. For it we have calculated values of $J_{z}, J_{+}$and $J_{-}$(these values come out in the BIREC computer program). The biggest inter-doublet transition $\Gamma_{7}-\Gamma_{9}^{(1)}$, denoted as excitation A in [8], is built up from $J_{+}=J_{-}=4.8554$. The biggest intra-doublet transition $\Gamma_{7}-\Gamma_{7}$, proceeded without a change of the energy in the paramagnetic state and with an energy change in the magnetically ordered state, is built up from $J_{+}=J_{-}=5$ and $J_{z}=0.5$. The calculated largest probabilities occur for the inter-doublet transitions $\Gamma_{7}-\Gamma_{9}^{(1)}, \Gamma_{9}^{(1)}-\Gamma_{8}^{(1)}$ and $\Gamma_{8}^{(1)}-\Gamma_{8}^{(2)}$ exactly as they have been revealed in the INS experiment of Donni et al. (the excitation A, B
TABLE I

Calculated probabilities $\left|\left\langle\Gamma_{i}\left|J_{\perp}\right| \Gamma_{j}\right\rangle\right|^{2}$ for the transitions between the CEF levels in the inelastic-neutronscattering on powder sample of $\mathrm{NdPd}_{2} \mathrm{Al}_{3}$.

\begin{tabular}{l|c|c|c|c|c}
\hline \hline$\left|\left\langle\Gamma_{i}\left|J_{\perp}\right| \Gamma_{j}\right\rangle\right|^{2}$ & & & & & \\
(calculated) & $\Gamma_{7}$ & $\Gamma_{9}^{(1)}$ & $\Gamma_{8}^{(1)}$ & $\Gamma_{9}^{(2)}$ & $\Gamma_{8}^{(2)}$ \\
\hline$\Gamma_{8}^{(2)}(142 \mathrm{~K})$ & & & & & 15.26 \\
$\Gamma_{9}^{(2)}(137 \mathrm{~K})$ & & & & 25.74 & 6.09 \\
$\Gamma_{8}^{(1)}(49 \mathrm{~K})$ & & & 8.59 & 0.05 & 11.07 \\
$\Gamma_{9}^{(1)}(9.7 \mathrm{~K})$ & & 2.59 & 13.29 & 0.83 & 0.57 \\
$\Gamma_{7}(0)$ & 17.00 & 15.72 & 0 & 0.28 & 0
\end{tabular}

and $\mathrm{C}$ in Ref. [8], respectively). It confirms that the derived electronic structure, with the attributed eigenfunctions, is correct with the very high certainty.

By means of our dynamical computer program [10] with these CEF parameters we have calculated properties of the magnetically-ordered state. With the intersublattice molecular-field coefficient $\lambda_{R R}=2.0 T / \mu_{\mathrm{B}}$ we have reproduced, by calculating the free energy, the relevant Néel temperature of $7.5 \mathrm{~K}$ (like in the experiment), but the ordered moment, being perpendicular to the $c$-axis like in the experiment, amounts at $0 \mathrm{~K}$ to $2.78 \mu_{\mathrm{B}}$. This value is much larger than the experimental value of $2.28 \mu_{\mathrm{B}}[11]$ - the deviation increases with the lowering temperature. One should remember that in our calculations we take energy levels as extremely thin energy lines which is rather unphysical in the energy scale below $0.2 \mathrm{meV}$. It would be desired to measure this local $\mathrm{Nd}$ moment by means of other local method like the hyperfine field in the Mössbauer-spectroscopy experiment. The substantial increase of the $\Gamma_{7}$ ground-state moment, in the paramagnetic state amounting to $1.82 \mu_{\mathrm{B}}$, is caused by a hybridization with the quite closely lying excited CEF states, the first lying at $9.7 \mathrm{~K}$, due to the effect of the internal magnetic (molecular) field. We have tried to modify slightly the CEF parameters, namely taking $B_{6}^{6}=4.0 \mathrm{mK}$, to increase slightly the energy of the first excited doublet, to $10.6 \mathrm{~K}$, but we have got practically the same results. From the physical point of view it is, however, important that the CEF calculations yield the formation of the magnetic order in the hexagonal plane.

By means of our dynamical computer program [10] we have obtained the fine electronic structure in the magnetically ordered state with splittings of the Kramers doublets, Fig. 2 and the temperature dependence of the heat capacity, Fig. 3 . In the $c(T)$ dependence the $\lambda$-type peak at $\mathrm{T}_{\mathrm{N}}$ is clearly visible. The derived molecular-field coefficient $\lambda_{R R}=2.0 T / \mu_{\mathrm{B}}$ yields the molecular field acting on the $\mathrm{Nd}$-ion at $0 \mathrm{~K}$ as $5.5 \mathrm{~T}$. It means that an external field of $10 \mathrm{~T}$ should produce the field-induced ferromagnetic state with a saturated magnetization of 2.3$2.8 \mu_{\mathrm{B}}$ /f.u. Moreover, we expect a non-linear increase of the magnetization with the field applied along the easy magnetic direction within the hexagonal plane like it has been observed in case of $\mathrm{Ho}_{2} \mathrm{Co}_{17}$ [12]. 
Discussing more the magnetic properties we mention that despite of much larger ordered moment the derived set of CEF parameters perfectly reproduces temperature dependence of the paramagnetic susceptibility for both main crystallographic directions derived experimentally on the single-crystalline sample [8]. It is worth noting that $a b$ initio calculations performed in Ref. [13], by means of the self-interaction corrected local spin-density approximation, were not able to reproduce these temperature dependences of the susceptibility as one can see inspecting Fig. 8 of Ref. [13].

We would like to point out that the good reproduction of experimental results of $\mathrm{NdPd}_{2} \mathrm{Al}_{3}$ confirms that all $\mathrm{Nd}$ atoms equally contribute to the magnetic susceptibility and to the heat capacity. It means that all $\mathrm{Nd}$ atoms have the same electronic structure as is shown in Figs. 2 and 3 .

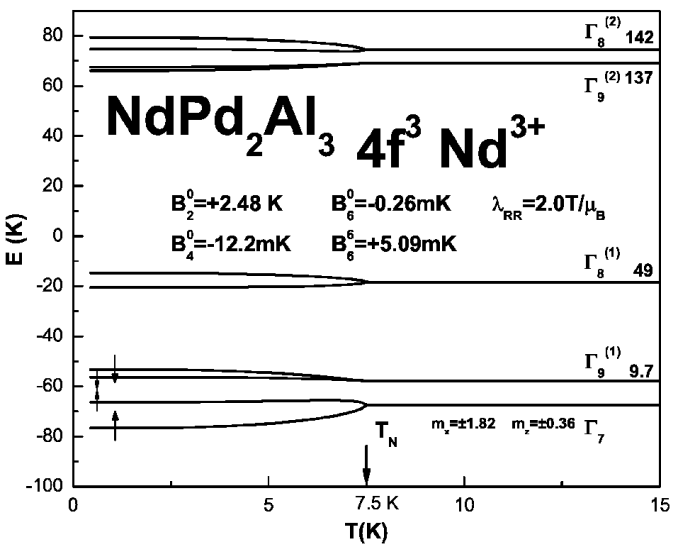

Fig. 2. Calculated fine electronic structure in the magnetic state. The appearance of the internal magnetic field below $T_{\mathrm{N}}$ causes the characteristic splittings of the Kramers doublets.

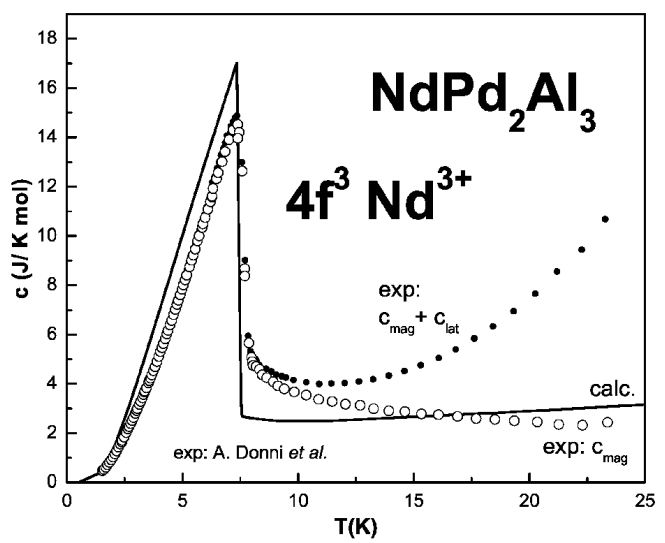

Fig. 3. Calculated temperature dependence of the heat capacity of $\mathrm{NdPd}_{2} \mathrm{Al}_{3}$ associated with the fine electronic structure of the $\mathrm{Nd}^{3+}$ ion. Experimental data after Ref. [11].
TABLE II

Calculated crystal-field parameters for isostructural and isoelectronic compounds $\mathrm{RPd}_{2} \mathrm{Al}_{3}(\mathrm{R}=\mathrm{Pr}, \mathrm{Ce}, \mathrm{Sm})$ calculated from parameters of $\mathrm{NdPd}_{2} \mathrm{Al}_{3}$ using relevant single-ion characteristics of Stevens and of the FreemanWatson radial moments $\left\langle r_{f}^{n}\right\rangle$ (in the atomic units of $a_{\mathrm{B}}^{n}$ ) tabulated in Table 2.4 in Ref. [15].

\begin{tabular}{l|c|c|c|c}
\hline \hline & $\mathrm{NdPd}_{2} \mathrm{Al}_{3}$ & $\mathrm{PrPd}_{2} \mathrm{Al}_{3}$ & $\mathrm{CePd}_{2} \mathrm{Al}_{3}$ & $\mathrm{SmPd}_{2} \mathrm{Al}_{3}$ \\
\hline$\left\langle r^{2}\right\rangle$ & 1.114 & 1.208 & 1.309 & 0.9743 \\
$\left\langle r^{4}\right\rangle$ & 2.910 & 3.396 & 3.946 & 2.260 \\
$\left\langle r^{6}\right\rangle$ & 15.03 & 18.72 & 23.31 & 10.55 \\
\hline $\mathrm{B}_{2}^{0}[\mathrm{~K}]$ & +2.48 & +8.85 & +25.9 & -13.9 \\
$\mathrm{~B}_{4}^{0}[\mathrm{mK}]$ & -12.2 & -35.9 & +362 & +81.4 \\
$\mathrm{~B}_{6}^{0}[\mathrm{mK}]$ & -0.26 & +0.52 & & \\
$\mathrm{~B}_{6}^{6}[\mathrm{mK}]$ & +5.1 & -10.18 & &
\end{tabular}

\section{2 $\mathrm{RPd}_{2} \mathrm{Al}_{3}(\mathrm{R}=\mathrm{Pr}, \mathrm{Ce}$ and $\mathrm{Sm})$}

Having established CEF parameters for $\mathrm{NdPd}_{2} \mathrm{Al}_{3}$ we have evaluated, by means of the single-ion assumption, the $\mathrm{CEF}$ parameters for other $\mathrm{RPd}_{2} \mathrm{Al}_{3}$ compounds. We recalculate $\mathrm{CEF}$ parameters from $\mathrm{NdPd}_{2} \mathrm{Al}_{3}$ to isostructural compounds for $\mathrm{R}=\mathrm{Ce}$, Pr and $\mathrm{Sm}$, using the singleion theory, i.e. making use of the Stevens coefficients and the relevant $f$-radial moments, $\left\langle r_{f}^{n}\right\rangle$, the $f$-electron radius in power of $n$. These parameters are collected in Table II.

Years ago we recalculate $\mathrm{CEF}$ parameters from $\mathrm{UPd}_{2} \mathrm{Al}_{3}$ to another actinide compound $\mathrm{NpPd}_{2} \mathrm{Al}_{3}[9]$ explaining within the crystal-field model the experimentally observed magnetic properties of $\mathrm{NpPd}_{2} \mathrm{Al}_{3}$, in particular the $\mathrm{Np}^{3+}$ moment value and the moment direction to be along the hexagonal $c$ axis, i.e. perpendicularly to the uranium moment in $\mathrm{UPd}_{2} \mathrm{Al}_{3}$. The same single-ion crystal-field mechanism works in case of $\mathrm{NpGa}_{2}$ compared to $\mathrm{UGa}_{2}[14]$.

The derived electronic structure for $\mathrm{RPd}_{2} \mathrm{Al}_{3}$ with $\mathrm{R}=$ $\mathrm{Ce}, \mathrm{Pr}$, and $\mathrm{Sm}$, predicts the nonmagnetic ground state $\Gamma_{1}$ for $\operatorname{PrPd}_{2} \mathrm{Al}_{3}$ and the magnetic ground state for the $\mathrm{Ce}$ and $\mathrm{Sm}$ compounds. For $\mathrm{SmPd}_{2} \mathrm{Al}_{3}$ the easy magnetic direction is along the $c$-axis i.e. perpendicularly to the neodymium moment in $\mathrm{NdPd}_{2} \mathrm{Al}_{3}$. These predictive results are quite similar to those in Ref. [8]. It confirms that our computer code uses, for instance, the same theoretical values of the radial moments as theoretically derived years ago by Freeman and Watson and collected by us in Table 2.4 of Ref. [15]. Even such simple fact can be a reason for obtaining different results in different $a b$ initio calculations of CEF parameters. In Ref. [13] the authors have tabulated the radial moments used by them for the $\mathrm{Pr}^{3+}$ and $\mathrm{Nd}^{3+}$ ions. For the $\mathrm{Nd}^{3+}$ ion in $\mathrm{NdPd}_{2} \mathrm{Al}_{3}$ they used relevant radial moments as $1.33 a_{\mathrm{B}}^{2}, 6.07 a_{\mathrm{B}}^{4}$ and $87.5 a_{\mathrm{B}}^{6}$. These values are substantially larger than values of $1.114 a_{\mathrm{B}}^{2}, 2.910 a_{\mathrm{B}}^{4}$ and $15.03 a_{\mathrm{B}}^{6}$ used by our group and the group of Prof. Furrer. Thanks reporting by the authors of Ref. [13] these radial moments the progress in getting agreement between different theoretical descriptions can be reached - usually theoretical $a b$ initio papers do not provide such basic input/outcome 
results. The same reproach to presentation of theoretical $a b$ initio results is that authors do not show the resultant charge distribution which is astonishing because the charge distribution is the basic ingredient/outcome of the $a b$ initio calculations. Without giving the charge distribution any discussion about calculations of the crystalfield parameters is impossible. We note that the positive value of the leading second-order CEF parameter $\mathrm{B}_{2}^{0}$ and a quite close value to that shown in Table II can be obtained using the point-charge model, i.e. within the ordinary really first-principles calculations making use of the ordinary electrostatic origin of the CEF parameters, taking into account only the 8 nearest $\mathrm{Nd}^{3+}$ neighbors (six $\mathrm{Nd}^{3+}$ ions in the hexagonal plane and two apical ions).

Finally, we have compared the derived CEF parameters of $\mathrm{NdPd}_{2} \mathrm{Al}_{3}$ with those derived for isostructural compounds $\mathrm{UPd}_{2} \mathrm{Al}_{3}(5.3 \mathrm{~K},+40 \mathrm{mK},-0.02 \mathrm{mK}$ and $-26 \mathrm{mK}$, respectively) [7] and $\mathrm{NdNi}_{5}(+3.35 \mathrm{~K}$, $+14.5 \mathrm{mK},-0.35 \mathrm{mK}$ and $-13.5 \mathrm{mK}$, respectively) [16]. This latter compound can be considered as $\mathrm{NdNi}_{2} \mathrm{Ni}_{3}$. The derived-at-present CEF parameters of these different compounds are quite close. In fact, a systematic larger value for the $\mathrm{U}$ compound is expected compared to $\mathrm{Nd}$ compounds. One may would expect the perfect similarities but we consider already these similarities as revealing the beauty of the solid-state physics at the atomic level taking into account that we work here with the electronic structure in the meV energy scale. Let us note that standard $a b$ initio band structure calculations work with the electronic structure in the eV energy scale, i.e. with 1000 times smaller accuracy.

\section{Conclusions}

Magnetic and electronic properties of $\mathrm{NdPd}_{2} \mathrm{Al}_{3}$ have been nicely described with the $\mathrm{Nd}^{3+}$ ions within the crystal-field theory revealing that all $\mathrm{Nd}$ atoms equally contribute to the temperature dependence of the paramagnetic susceptibility and the heat capacity. We consistently described, within the quantum atomistic solid state theory, both the paramagnetic and the magnetic state. The fine electronic structure of the $\mathrm{Nd}^{3+}$ ion nicely correlates with the electronic structure of the $\mathrm{U}^{3+}$ ion in $\mathrm{UPd}_{2} \mathrm{Al}_{3}$ giving strong evidence for the trivalent uranium state in the isostructural heavy-fermion superconductor $\mathrm{UPd}_{2} \mathrm{Al}_{3}$. These studies indicate that physically adequate theoretical description has to be made at the atomic scale and at the meV energy scale.

\section{References}

[1] K. Ghost, S. Ramakrishnan, S.K. Malik, G. Chandra, Phys. Rev. B 48, 6249 (1993).

[2] C. Geibel, C. Schank, S. Thies, H. Kitazawa, C.D. Bredl, A. Bohm, M. Rau, A. Grauel, R. Caspary, R. Helfrich, U. Ahlheim, G. Weber, F. Steglich, Z. Phys. B Condens. Matter 84, 1 (1991).

[3] C. Grauel, A. Bohm, H. Fisher, C. Geibel, R. Kohler, R. Modler, C. Schank, F. Steglich, G. Weber, T. Komatsubara, N. Sato, Phys. Rev. B 46, 5818 (1992).

[4] N.K. Sato, N. Aso, K. Miyako, R. Shiina, P. Thalmeier, G. Vareloglannis, C. Geibel, F. Steglich, P. Fulde, T. Komatsubara, Nature 410, 340 (2001).

[5] L.M. Sandratskii, J. Kubler, P. Zahn, I. Mertig, Phys. Rev. B 50, 15834 (1994); K. Knopfle, A. Mavromaras, L.M. Sandratskii, J. Kubler, J. Phys. Condens. Matter 8, 901 (1996).

[6] G. Zwicknagl, A. Yaresko, P. Fulde, Phys. Rev. B 68, 052508 (2003); Phys. Rev. B 65, 081103(R) (2002); P. McHale, P. Fulde, P. Thalmeier, Phys. Rev. B 70, 014513 (2004).

[7] R.J. Radwanski, Z. Ropka, Czech. J. Phys. 54, D295 (2004); Acta Physica 2, 14 (2007).

[8] A. Donni, A. Furrer, H. Kitazawa, M. Zolliker, J. Phys., Condens. Matter 9, 5921 (1997).

[9] R.J. Radwanski, R. Michalski, Z. Ropka, Acta Phys. Pol. B 31, 3079 (2000).

[10] R.J. Radwanski, N.H. Kim-Ngan, F.E. Kayzel, J.J.M. Franse, D. Gignoux, D. Schmitt, F.Y. Zhang, J. Phys. Condens. Matter 4, 8853 (1992); R.J. Radwanski, R. Michalski, Z. Ropka, A. Blaut, Physica B 319, 78 (2002).

[11] A. Donni, H. Kitazawa, P. Fischer, T. Vogt, A. Matsushita, Y. Iimura, M. Zolliker, J. Solid State Chem. 127, 169 (1996).

[12] R.J. Radwanski, J.J.M. Franse, Physica B 154, 181 (1989).

[13] Z.S. Liu, M. Richter, M. Divis, H. Eschring, Phys. Rev. B 60, 7981 (1999).

[14] R.J. Radwanski, J. Phys. Condens. Matter 8, 10467 (1996).

[15] J.J.M. Franse, R.J. Radwanski, Handbook of Magnetic Materials, Vol. 7, Eds. K.H.J. Buschow, Elsevier, North-Holland 1993, p. 307.

[16] N.-T.H. Kim-Ngan, R.J. Radwanski, F.E. Kayzel, J.J.M. Franse, J. Magn. Magn. Mater. 157-158, 407 (1996). 